\title{
Autorenfassung: Vernunft diesseits von Zwecken und Mitteln
}

Erstveröffentlicht in: Internationales Jahrbuch des Deutschen Idealismus, Heft 1 2003, hrsg. V. Karl Americks und Jürgen

Stolzenberg, S. 43-56

\section{Hans Friedrich Fulda}

2003/2014 
Inhalt

1. Vorüberlegungen zum Thema

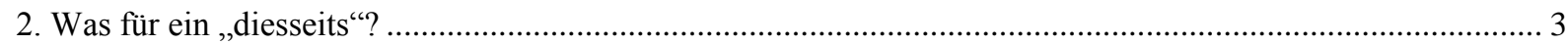

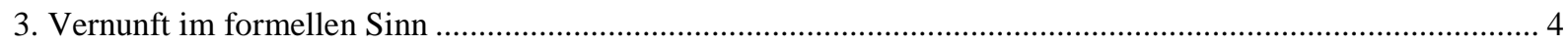

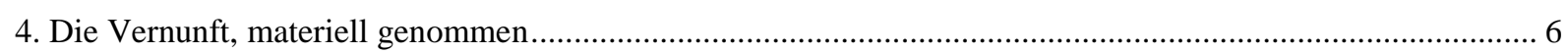

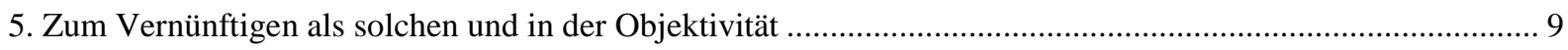

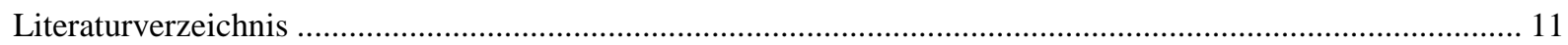

$\{\mid$ S. 43$\}$

Das Substantiv ,Vernunft‘ ist ein Rätselwort: Kein substantivierter Infinitiv und doch wie ein solcher schlechterdings nicht in den Plural zu setzen. Der Begriff hingegen, den das Wort seit langem bezeichnet, hat so abenteuerliche Schicksale erlitten, daß ihm terminologiegeschichtlich kaum beizukommen ist. Man vergleiche unsere Wörterbücher philosophischer Begriffe und denke daran, daß das Nomen, Vernunft ${ }^{`}$ sowohl zur Übersetzung des griechischen Begriffsworts ,voữ $\varsigma^{`}$ herangezogen wird (und taugt), als auch herhalten muß zur Übersetzung von ,ratio‘, das seinerseits im Lateinischen seit Cicero zur Übersetzung von , $\lambda$ ó $\varsigma^{`} \varsigma^{`}$ dient, während ,voư $\varsigma^{‘}$ mit ,intellectus ‘ übersetzt wird, für das sich im Deutschen früh ,Verstand` eingebürgert hat — auch so ein nicht in den Plural setzbares Substantiv mit schwer zu fassender Bedeutung. Man könnte darüber tiefsinnig werden. Oder man zieht es neudeutsch-nüchtern vor, den Ausdruck, Vernunft ‘ und seine grammatischen derivativa zugunsten von ,Ratio“ oder ,Rationalität‘ zu meiden. Aber da uns Konzepte im Deutschen Idealismus beschäftigen sollen, will mir dieses Surrogat nicht recht schmecken. Ich werde es verschmähen und mich stattdessen zunächst an unser Eigenschaftswort, vernünftig ' halten. Das hat zumindest den Vorteil, daß wir von einigen unverfänglichen Verwendungsweisen ausgehen können, wenn wir sagen möchten, was unter, Vernunft‘ zu verstehen ist. $\{\mid$ S. 44$\}$

\section{Vorüberlegungen zum Thema}

Man bezeichnet Erwägungen, Ratschläge, Pläne, Entschlüsse oder sie ausführende Handlungen bzw. die Personen oder Einrichtungen, die sie ausführen, exemplarischerweise als vernünftig, wenn sie im Sinn eines guten Lebens, das seine Prioritäten richtig setzt und beachtet, klug sind. Dementsprechend wäre Vernunft, welche jemand (oder eine Institution) besitzt, sozusagen die $\tilde{\eta} \xi 1 \varsigma$ des Klugen, der Inbegriff oder die Grundlage seiner dianoetischen Tugenden. Ich bin überzeugt, daß Vernunft nicht zuletzt so etwas ist. Aber erschöpft diese Auffassung den anspruchsvoll tiefen Sinn des dunklen Wortes ,Vernunft‘? Mindestens drei Einwände sprechen dagegen:

1. Das Argument der offenen Frage: Etwas herbeizuführen, das besser als anderes dem erfüllten Leben dient, dürfte klug sein. Aber ist es damit eo ipso auch vernünftig? Ist es das z. B. auch noch, wenn es darum geht, in der richtigen Weise zu sterben? Mag es nicht Forderungen praktischer Vernunft geben, die von denen der Klugheit verschieden und ihnen sogar übergeordnet sind, wie z. B. die Forderung der Gerechtigkeit?

2. Das Argument der Kontextualität von Klugheit, wie ich es nennen möchte: Etwas ist klug im Kontext eines erfüllten Lebens. Dessen komplexes Konzept ist der Zuschreibung von Vernünftigkeit, die mit Klugheit 
einhergeht, vorauszusetzen. Entsprechend mag es mehrere, auf die verschiedenen Entwürfe solchen Lebens relative Klugheiten geben; das Wort ,Klugheit‘ widersetzt sich dieser Pluralisierung nicht. Anders das singulare tantum , Vernunft'.

3. Das Argument zu großer Spezifizität: Vernunft als $\tilde{\eta} \xi 1 \varsigma$ des Klugen trifft bestenfalls den Sinn praktischer Vernunft. Was aber ist Mit der theoretischen Vernunft bzw. mit einer Auffassung von der Einen Vernunft, die der Spezifikation in eine theoretische und eine praktische noch vorausgeht? Ihr kann die Identifikation des Klugen mit dem Vernünftigen nicht gerecht werden. Was dann?

Die Suche nach begrifflicher Bestimmung der Einen Vernunft verlangt, von den lebensweltlichen Konkretionen abzusehen, die wir beim Identifizieren des Klugen und Vernünftigen vor Augen haben, und nach einem abstrakteren Begriff von Vernunft Ausschau zu halten. Das Absehen nicht nur von diesem oder jenem Konzept erfüllten Lebens, sondern sogar vom Unterschied der praktischen und der theoretischen Vernunft wird uns dabei nahelegen, Charakteristika für das generell Vernünftige im Verhältnis von Zwecken und Mitteln zu vermuten. Denn auch mit unserer theoretischen Vernunft gehen wir jedenfalls auf Zwecke aus (der wahren und Sach-adäquaten Erkenntnis nämlich) und mobilisieren wir Mittel, sie zu verwirklichen. So mag man ein einheitliches, aber leicht in verschiedene Richtungen spezifizierbares Merkmal der Vernunft darin erblicken, daß in zwei symmetrisch zueinander gehö- $\{\mid$ S. 45\} renden Verhältnissen von Mitteln und Zwecken bestimmte Bedingungen erfüllt sind: die Bedingung, daß Mittel für (ihrem Gebrauch vorausgesetzte) Zwecke zur Verwirklichung dieser Zwecke auch tatsächlich taugen; und die Bedingung, daß etwas, das zum Zweck gemacht wird, auch von gegebenen oder mobilisierbaren Mitteln aus erreichbar ist.

Doch diese formelle Bestimmung von Vernünftigem, die uns immerhin Merkmale eines genereller applikablen Vernunfbegriffs liefert, vermag dessen Gehalt ebenfalls nicht auszuschöpfen. Dafür sprechen mindestens zwei Argumente:

1. Das Argument bloßer Relativität: Die genannten Bedingungen zeichnen etwas als vernünftig aus nur relativ zu anderem, dessen Vernünftigkeit dabei bereits vorausgesetzt werden muß: das Mittel nämlich relativ zu einem Zweck, bei dem Vernunft bereits unterstellt ist; bzw. den Zweck relativ zu einem Mittelvorrat unter der Voraussetzung, daß im Gebrauch von Mitteln aus diesem Vorrat Vernunft enthalten ist.

2. Das Argument begrifflicher Abgeleitetheit: Daß etwas durch Erfüllung gewisser Bedingungen „vernünftig“ sei, heißt nur, daß es an Vernunft partizipiert, während es gerade nicht diese Vernunft selber ist oder ausmacht. Die Berechtigung, es als vernünftig zu bezeichnen, hätte sich allererst aus dem reicheren begrifflichen Gehalt eines „substantiellen“ Vernunfbegriffs zu ergeben. Wenn bei der Auskunft über Vernunft die vorgeschlagene formelle Bestimmung des Vernünftigen diesen Begriff ersetzen soll, so gibt sie uns sozusagen Steine statt Brot, wenn auch vermischt mit ein paar Brotkrumen.

Fazit dieser Vorüberlegungen: Es mag so sein, daß Vernunft vorab an dem zutage tritt, was wir mit Überzeugung vernünftig nennen; und das so zu nennende „Vernünftige“ mag seinen paradigmatischen Ort im Bereich unseres gemeinschaftlichen, recht geführten Lebens haben. Jenseits davon (in der außermenschlichen Natur oder sogar jenseits von der, wenn es ein solches Jenseits gibt) dürfte es jedenfalls, wenn überhaupt, noch viel schwerer zu fassen sein. Aber das Vernünftige gibt sich dem Versuch, es allgemein genug zu denken, auch nicht einfach im alltäglichen Kontext seines Auftretens zu erkennen. Was Vernunft substantiell ausmacht, scheint sich diesem Kontext sogar systematisch zu entziehen. Wenn überhaupt, wird man es wohl nur diesseits von Zwecken und Mitteln zu fassen bekommen. 


\section{Was für ein „diesseits“?}

Es liegt nahe zu sagen, daß wir in uns selbst zurückgehen müssen, wenn wir uns über ,Vernunft' in einer grundlegenden Bedeutung dieses Worts aufklären wollen. Spätestens seit Descartes war ein Rückgang in dieses „Selbst“ das $\{\mid$ S. 46\} große Versprechen: auf jenes ursprünglich Eine zu stoßen, das anscheinend die Vernunft selber ist und worin sich die Gründe dafür entdecken lassen, daß menschliche Wesen wie ich der Erkenntnis fähig sind — nicht zuletzt beim Gebrauch von Mitteln und bei einer mir selbst gemäßen Systematisierung von Zwecken. Allenfalls in diesem Einen werden wir vor die Differenz des theoretisch und des praktisch Vernünftigen gelangen. Allenfalls von da aus können wir uns auch Hoffnung machen, ein Problem zu lösen, das sich erst jetzt abzeichnet: die Schwierigkeit, uns begreiflich zu machen, wie Mittel (als bestehende oder herzustellende Zustände und Ereignisse in der objektiven Wirklichkeit) überhaupt dazu dienen können, Zwecke, wie man sagt, zu „realisieren“, wo Zwecke doch etwas vom Ursprung her ganz anderes sind als jene Zustände und Ereignisse: konzeptuell und von unsereinem entworfen, also subjektiven Ursprungs. Warum sind Zwecke angesichts der Heterogenität von Zwecken und Mitteln nicht dazu verdammt, die Erwartungen, die wir mit ihnen haben, beim Versuch der Realisierung zu frustrieren? Man braucht nicht mit Descartes die Lehre von zweierlei Substanzen zu teilen, um dieses Problem zu haben. Spinoza hatte es ebenfalls — und hat es durch den Gewaltstreich zu beseitigen versucht, alle Teleologie in den Bereich bloß irrigen Meinens zu verbannen. Aber wenn man sich (zumindest) um der praktischen Vernunft willen dazu nicht verstehen kann: droht dann nicht für das Realisieren von Zwecken der infinite Regreß, daß jeder vermeintlich erreichte Zweck auch nur ein Mittel ist, oder kommt es gar zu einem derart hoffnungslosen Auseinanderklaffen von Zwecken und Mitteln, daß keine Kombination von Mitteln tauglich scheint, einen Zweck zu erfüllen? Hinter all dem aber lauert eine noch größere Frage: Wie können wir überhaupt unter „Seiendem“, das exemplarisch ist, nämlich objektiv der Fall ist, Zwecke und Mittel auszeichnen, die doch beide zunächst etwas Subjektives, nämlich mit unserer „Subjektivität“ Zusammenhängendes sind; und wie können wir dann zwischen ihnen beiden differenzieren und sie der Vernunft entsprechend aufeinander bezogen denken in dem Objektiven, worin wir etwas als Mittel für Weiteres (als einen erreichten Zweck) denken und worin wir zwischen beiden ein vernünftiges Verhältnis konstatieren wollen?

Mehr noch als die vorgebrachten Argumente geben uns diese Fragen wohl Anlaß, die Vernunft zunächst einmal diesseits von Zwecken und Mitteln zum Thema zu machen und dazu auf uns selbst zurückzukommen. Aber was an allem, das mehr als Zwecke und Mittel zu „uns“ gehört, kann uns über Vernunft und Vernünftiges besser aufklären helfen als unser Urteil über Zusammenhänge von Mitteln und Zwecken? Etwa Meinungen, Einstellungen, Kompetenzen, Präferenzen, aus denen wir handeln und fürs Handeln Zwecke setzen sowie über Mittel zu Zwecken disponieren? Das ist ebenso unwahrscheinlich, wie es dem ,linguistic turn“ in der Philosophie, insbesondere aber in der philosophischen Psychologie, zuwider wäre. Denn über so schlüpfrige subjektive Zustände, wie z. B. Meinungen sind, und über ihre $\{\mid$ S. 47 $\}$ angemessene Beschreibung wissen wir am besten von jenem „Verhalten“ aus Bescheid, das — wie insbesondere das sprachliche Verhalten — für jedermann „,von außen“ her am besten zugänglich ist und nur zusätzlich hierzu für den, der sich in einem jener Zustände befindet, auch „,von innen“ her (z. B. im Hinblick auf die Frage, wie es ist, in einem solchen Zustand zu sein). Außerdem: Vernunft im Verhältnis von Mitteln und Zwecken ist Vernunft in etwas Objektivem, also gerade nicht bloß Vernunft in der Subjektivität miteinander kooperierender geistiger Tätigkeiten und koordinierter Vermögen. Allein aus Einsicht in solch einseitige Subjektivität dürften der Übergang zur Objektivität und die Vernunft in dieser Objektivität nicht begreiflich zu machen sein. Der Weg der Abstraktion 
und sprachlichen Formalisierung, welcher schon mit der Rede von Zwecken und Mitteln eingeschlagen ist, muß also wohl noch ein ganzes Stück weitergegangen werden, um uns auf einen Begriff von Vernunft und Vernünftigem zu bringen, der greifbar ist — und doch so umfassend, daß er nicht in die Schwierigkeiten hineintreibt, die sich einstellen, wenn man die Struktur der Vernunft an bloß relativ Vernünftigem aufdecken möchte. Der Weg muß im Zurückkommen auf uns selbst so weit gegangen werden, daß er nicht nur die begriffliche Differenz von theoretischer und praktischer Vernunft, sondern auch diejenige von subjektiver und objektiver Vernunft unterschreitet. So wird am Ende dieses Wegs ein Begriff von Vernunft als von etwas blo $\beta$ Formellem stehen, das geistige Tätigkeiten ebenso auszeichnet wie dasjenige, womit sie sich befassen. Von diesem Formellen wird dann die Frage sein, ob ihm auch ein Begriff von Vernunft, materiell genommen, entspricht, d. h. einer, der die Vernunft selbst beschreibt, — und ob innerhalb solcher Beschreibung auch begriffen werden kann, was das Vernünftige als solches ausmacht. Das wiederum mag die Frage veranlassen, ob von da aus auch ein Begriff von Vernunft, objektiv genommen, oder vom Vernünftigen in der Objektivität dergestalt entwickelt werden kann, daß dazu auch ein bestimmtes Verhältnis von Zwecken und Mitteln gehört. Mit diesem Überlegungsgang jedenfalls möchte ich mich nun dem Vernunftbegriff im Deutschen Idealismus annähern. Das „diesseits“ (von Mitteln und Zwecken) ist also dasjenige, was meinem Überlegungsgang in allen seinen Schritten mit Ausnahme des letzten zugrundeliegt und womit diese Schritte sich zu befassen haben.

\section{Vernunft im formellen Sinn}

Die Deutschen Idealisten haben sich um Aufdeckung und Präzisierung eines universalen Vernunftbegriffs bemüht. Ihre Bemühungen hätten wohl erheblich an Überzeugungskraft gewonnen, wenn nicht die Frage, was Vernunft im menschlichen Forschen generell ist, weitgehend ausgespart geblieben wäre — als Frage nämlich

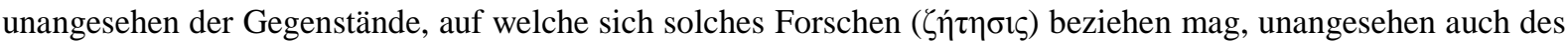
Unterschieds einer $\{\mid$ S. 48 $\}$ Ausrichtung auf theoretische oder praktische Erkenntnis, ja sogar unangesehen des Unterschieds, den es macht, ob die Aussagen, die beim Forschen im Spiel und zu erwägen sind, überhaupt einen Referenten haben, den man als Gegenstand von (theoretischer oder praktischer) Erkenntnis betrachten darf, oder ob sie keine solche Referenz besitzen. Von Vernunft handeln, dabei aber von all diesen Unterschieden absehen, heißt, die Vernunft in formellem Sinn nehmen. Was aber bleibt dann übrig?

Das kann man sich, denke ich, anhand von Ch. S. Peirce's Analyse des Schlußfolgerungsprozesses (,reasoning“) klarmachen, wenn man beachtet, daß es bei diesem Prozeß nicht um die Frage geht, was „wir“ (als Schlußfolgernde) zu tun oder zu lassen haben, und daß es dabei nicht eo ipso schon einer kontrolliert eingesetzten Empirie bedarf, wie Peirce allerdings vorschnell annimmt. Vielmehr haben wir uns in der Analyse nur um die Gründe zu kümmern, die logisch (also vernünftigerweise) berechtigen, von einer Aussage oder Aussagenkombination zu einer anderen und von $\mathrm{ihr} z u$ wieder anderen fortzugehen, schließlich aber auch den Prozeß zu beenden und ihn in einer Aussage zur Ruhe kommen zu lassen. Die Gründe solcher Berechtigung sind zugleich (partielle) Gründe für die Wahrheit derjenigen Aussagen, zu denen berechtigtermaßen übergegangen wird. Denn das in letzter Instanz Berechtigende ist das im Prozeß zu erforschende Wahre. Der Prozeß des berechtigten Übergehens von Aussage zu Aussage läßt sich also genausogut beschreiben als einer des Wahren, das sich im Prozeß von irgendeinem insoweit kontingenten, bedingten Ausgangspunkt aus zu erkennen gibt. Beide Beschreibungen laufen in der Sache aufs selbe hinaus. Auf den Unterschied zwischen der einen, den 
„subjektiven“ Aspekt betonenden, und der anderen, die den „objektiven“ Aspekt (des Wahren) in Betracht zieht, kommt es dann nicht mehr an.

Unter einem wichtigen, altbekannten Teilaspekt hat man es beim umrissenen Prozeß und seinen Gründen mit einem deduktiven Schluß zu tun, paradigmatisch mit einem direkten Beweis. Ein solcher Schluß ist höchst eindrucksvoll, weil er zu einer uneingeschränkt als wahr zu bewertenden Conclusio führt, wenn der Beweis gelingt. Er überträgt dann die Wahrheit seiner Prämissen auf die Conclusio. Aber da dies nur für den Fall des gelingenden Beweises gilt, erhebt sich sogleich die Frage, unter welchen die Prämissen betreffenden Voraussetzungen die Möglichkeit eines gelingenden Beweises steht. Die Antwort ist, in Kürze, daß die Prämissen selbst wahr sein, aber auch die für eine mögliche Formalisierung des Schlusses erforderliche Bestimmtheit hinsichtlich Aussagenverknüpfungen sowie hinsichtlich Prädikation und einer Referenz haben müssen, die bis zur Vereinzelung bestimmt ist oder wenigstens nur unbestimmt innerhalb eines bestimmten Individuenbereichs. Diese Antwort zieht natürlich die Frage nach sich, wie die Prämissen Wahrheit sowie Bestimmtheit erlangen und wie man sich vergewissern kann, daß sie beides besitzen. Eine alte Auskunft hierüber ist, daß wir wenigstens für gewisse, höchste Prämissen ein entsprechend hoch zu schätzendes Vermö- $\{\mid$ S. 49\} gen (,Vernunft‘ oder auch ,intuitiver Verstand“ genannt) haben und dadurch die Wahrheit sowie Bestimmtheit dieser Prämissen unmittelbar erfassen (oder „berühren“). Aber das ist eine Verlegenheitsauskunft, die nicht überzeugt. Denn gerade des Allgemeinen und nicht mehr Vagen, sondern präzis Bestimmten sind wir nicht unmittelbar teilhaftig, sondern allenfalls an einem Ende der „Bewegung“, welche das Forschen ist. Der Begriff der Vernunft als eines intuitiven Verstandes, welcher die Prämissen für deduktives Schließen aufnimmt, setzt einen Teil dessen, was formaliter zur Vernunft gehört, an die Stelle des Ganzen, das diese ist, und mißdeutet den Teil damit gründlich. Außerdem übergeht er, daß Vernunft nicht nur am Anfang, sondern auch am Ende des Prozesses, der sie ist, als dessen Grenze das Gegenteil von Bewegung, also Ruhe, enthält. Auf beide Grenzen hin muß der Prozeß in die Aussagen Wahrheit und Bestimmtheit hinein bringen, wenn er in formeller Hinsicht die Vernunft selber sein soll. Er muß also noch unter anderem Aspekt betrachtet werden als unter dem Teilaspekt deduktiver Schlußfolge. Und das so zu Betrachtende muß dem Beginn dieser Schlußfolge im Prozeß sogar vorhergehen.

Was für ein anderer Aspekt kann das sein — oder welche Mehrheit anderer Aspekte? Jedenfalls muß nach dem gegebenen Umriß des Prozesses nicht nur dessen Ende, sondern auch der Anfang möglichen deduktiven Folgerns vom Prozeß selber abhängig sein. Das letztere jedoch in doppelter Weise: Zum einen so, daß bereits vorhandene, aber problematische Annahmen die Glaubwürdigkeit, wahr zu sein, im Prozeß erlangen, und gleichfalls die Bestimmtheit, welche für ihre Tauglichkeit zu Prämissen direkter Beweise erforderlich ist. Zum andern aber muß der Prozeß (unter einem weiteren Aspekt), soweit solche Annahmen noch nicht vorliegen oder untereinander konkurrieren, vorab zu ihnen hinführen. Die Verfahren, die bezüglich empirischer Annahmen unter diesen beiden Gesichtspunkten erforderlich sind, hat Peirce diejenigen der Induktion und Abduktion genannt. Man muß prüfen, ob Gründe für das Übergehen (zu präziseren sowie für wahr gehaltenen oder zu ganz neuen Annahmen) dieses Übergehen auch schon berechtigen können, wenn die spezifischen Bedingungen für empirische Annahmen (und den Umgang mit ihnen) nicht gegeben sind oder von ihnen abgesehen wird.

Das Ergebnis der Prüfung fällt, glaube ich, bejahend aus: In der ersten Fallgruppe nämlich, wenn man diese in der nötigen Abstraktion denkt, handelt es sich um den Schluß von einer vorliegenden, aber vagen Annahme auf ihre rebus stantibus bestmögliche Bestimmtheit und Modalität dafür, daß sie als Prinzip deduktiver Folgerung in Frage kommt Der Schluß, d. h. Übergang, ist logisch berechtigt, weil er — durchgängig angewandt 
auf das Problem der Wahrheit und erforderlichen Bestimmtheit von Annahmen — „in the long run“ die Konvergenz bloßer Annahmen zum Wahren hin herbeiführen muß. Was dabei den Ausgangszustand der Annahme mit deren Endzustand vermittelt, muß nicht (wie bei der Induktion) eine Serie empirischer Beobachtungen $\{\mid$ S. 50 oder ein in unserer Erfahrungswelt angestelltes Experiment sein. Es kann auch ein Gedankenexperiment sein, wenn es nur der Bedingung genügt, daß man mit ihm nicht nach Übereinstimmung und Bestätigung Ausschau hält, sondern nach Widerlegung und daß man sich, wenn man es anstellt, der Resultate nicht schon im vorhinein vergewissert hat.

Das abstrakte Pendant zur Abduktion schließlich — der eigentlich synthetische Schritt im Prozeß — ist der Schluß auf einen rebus stantibus bestmöglichen Ausgangspunkt für Prinzipien zu eventuellen deduktiven Folgerungen bzw. für die Präzisierung und Beglaubigung von Annahmen. Der Schritt ist logisch berechtigt, weil einzig er Aussicht eröffnet, sich im Zusammenspiel der Gründe, die zu Schlußfolgerungen der anderen beiden Arten berechtigen, allmählich der Wahrheit anzunähern. Die Weise, den Schritt zu vollziehen, muß in einer Logik, die speziellere Aufgaben in Angriff nimmt, konkretisiert werden. Auch im Gebrauch-machen von dieser Logik aber bedarf es nicht eo ipso der Empirie. Grundsätzlich genügt es, daß man beim Pendant zur Abduktion von demjenigen seinen Ausgang nimmt, was kontra-intuitiv ist, und zu einer Annahme übergeht, die das Verwunderung Erregende verständlich macht und sich ihrerseits doch möglichst gut ins schon plausibel Gewordene einfügt; oder die, anders gesagt, am schon plausibel Gewordenen dessen Perspektive (auf deduktive Folgerungen hin) erneuert, aber an seinem begrifflichen Gehalt möglichst wenig Änderung vornimmt, um den Prozeß der Annäherung an Wahrheit nicht instabil zu machen.

Es leuchtet hoffentlich ohne Erläuterung ein, wenn ich behaupte, daß die drei Aspekte, unter denen Gründe bestehen, die den Übergang von Aussage zu Aussage berechtigen, sich zum Bild eines einzigen, einheitlichen Prozesses zusammenfügen und daß sich der Prozeß dabei als geeignet erweist, das Feld der in concreto vorliegenden Gründe für Übergänge sowohl nach oben, zum Allgemeineren hin, als auch nach unten, in

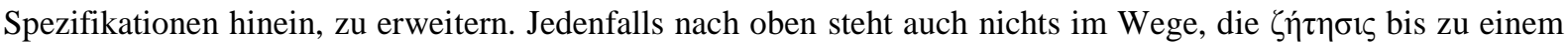
höchsten Allgemeinen auszudehnen, in welchem der Prozeß des Schlußfolgerns wenigstens unterm letztgenannten seiner Aspekte zur Ruhe kommt

Soviel kann man, glaube ich, über Vernunft im formellen Sinn und generell gesprochen sagen. Es ist darin nicht enthalten, daß der Prozeß, der die Vernunft in diesem Sinn ist, von uns mit einem gewissen Zweck durchlaufen werden muß. Es genügt, wenn man die in ihm enthaltenen Gründe berücksichtigt, die zum Übergang von Aussage zu Aussage berechtigen. Ferner: Wenn sich zeigen lassen würde, daß der (unterm letztgenannten Aspekt bezeichnete) eigentlich synthetische Schritt des Prozesses nicht nur nach oben führt, daß er vielmehr auch nach unten, zu einem Einzelnen führen kann, so dürfte man behaupten, die Bewegung schließe sich in gewisser Weise auch zu ihm (als dem Ende deduktiver Schlußfolgerung) hin ab. Das aber ist von der Vernunft im allgemeinen nur zu behaupten, wenn man sie materialiter nimmt. $\{\mid$ S. 51\}

\section{Die Vernunft, materiell genommen}

Der skizzierte Begriff formeller Vernunft ist der Begriff einer Prozessualität des Schlußfolgerns, die zu Wahrheit, Bestimmtheit und höchstmöglicher Allgemeinheit in Aussagen sowie darin enthaltenen Begriffen führt, - wobei „Schlußfolgern“ hier nur soviel heißt wie die logische Berechtigung, von einer Aussage (oder 
Aussagenverknüpfung) zu einer anderen überzugehen. Wer die dazu erforderlichen Abstraktionen oder wenigstens einige von ihnen aufhebt, ohne von Vernunft abzusehen, nimmt diese materiell. Diejenige der genannten Abstraktionen, die aufgehoben werden kann, ohne daß die Vernunft dabei gleich im objektiven Sinn genommen wird oder in einem hierauf relativen subjektiven Sinn, besteht im Berücksichtigen eines Referenten, der noch gar nicht Gegenstand im Sinn eines Objekts theoretischer oder praktischer Erkenntnis ist und auch nicht dessen subjektives Gegenstück als das spezifische Vermögen solcher Erkenntnis. Der diesen Gegenständen und Vermögen (in der Ordnung der Abstraktion) noch vorauszusetzende Referent ist die Vernunft selbst. Sie als solchen umfassendsten Referenten von Aussagen betrachten, heißt, sie materialiter zu nehmen. Zu ihr gehört somit alles, was über Vernunft im formellen Sinn gesagt wurde bzw. zu sagen wäre, soweit es nicht schon subjektive Tätigkeit oder Vermögen dazu betrifft, sondern bloß die Gründe, die zu dieser Tätigkeit und zum Gehalt von Aussagen berechtigen. Insofern ist der Übergang vom formellen zu einem allgemeinsten materiellen Konzept von Vernunft ganz einfach. Er ist bloß derjenige einer Aufhebung der Abstraktion bezüglich jenes Referenten von Aussagen, welcher die Vernunft selber ist (und nicht nur „das Wahre“ schlechthin). Was aber kommt durchs Aufheben dieser Abstraktion nun hinzu?

Ich denke, dazu kann man bereits mit Kant einiges sagen, ${ }^{1}$ wenn man nur den diesbezüglichen Kantischen Behauptungen ihre vermögenstheoretische Spezifikation benimmt. Zweifellos geht es bei den logisch berechtigenden Gründen letztlich um Prinzipien (während Kant die Vernunft bekanntlich als „Vermögen der Prinzipien“ bezeichnete) und geht es in diesen Prinzipien, wenn die Vernunft als ihr Referent ausgemacht ist, ausschließlich noch um begriffliche Gehalte sowie deren Bestimmtheit. Ferner sind Begriffe mit diesen Gehalten, wenn Begriffe von Vernunft an ihr selbst, höchstmögliche Prinzipien und Begriffe des Unbedingten (wie Kant die Vernunft als ein Vermögen des Unbedingten faßte); und die Begriffe sind als solche des Unbedingten gewiß auch Begriffe, die gewonnen werden durch Synthesis aus intellektuell gegebenen Elementen: aus Begriffen von Bedingtem nämlich, das bereits aller Erfahrung $\{\mid$ S. 52\} vorausgehend gedacht wird, dessen Begriffe aber in ihrer Anwendbarkeit bis zum Unbedingten erweitert werden. ${ }^{2}$ Nur sind sie in einer ihnen allen gewidmeten, speziellen Logik der Aufklärung über die Vernunft selbst zu untersuchen; und zu untersuchen nicht hinsichtlich der Gelegenheiten ihrer Entstehung (wie vage sie sich bei diesen auch bilden mögen), sondern hinsichtlich ihrer Bestimmtheit und Berechtigung — als Gehalte, die das Potential haben, die Vernunft in Beziehung auf sich selbst zu bestimmen. Das Verfahren, über sie aufzuklären, wird dahin führen müssen, sie zu thematisieren hinsichtlich ihres bestimmten, explizit zu machenden Inhalts sowie hinsichtlich der Form, gemäß der sie in der Selbstbestimmung der Vernunft kooperieren. Die Untersuchung, die nach diesem Verfahren anzustellen ist (worin immer es des Näheren bestehen mag), wird sich dabei, um eine kritische zu sein, zwischen Dogmatismus und Skeptizismus bewegen müssen. Sie muß Vorschläge der Prädikation, welche der Dogmatiker macht, der rigorosesten skeptischen Kritik unterwerfen, ohne im Skeptizismus zu münden. Soviel darf, glaube ich, mit Kant guten Gewissens behauptet werden.

\footnotetext{
${ }^{1}$ Es bahnt sich schon an in der Leibniz-Wolffischen Metaphysik. Vgl. z. B. Baumgarten, 1757, §§ 640 ff. — Näheres zum Zusammenhang mit dem Kantischen Vernunftbegriff wird ausgeführt in Fulda/Stolzenberg (Hrsg.), 2001, S. 11 ff.

${ }^{2}$ Man könnte einwenden, die Elemente seien doch als solche nicht schon Elemente der Vernunft. Sie seien jedenfalls so lange als ihr heterogen anzusehen, wie noch nicht ihr Ursprung aus der begrifflich bestimmten Vernunft erkannt ist; davor könnten sie nur als Elemente des Verstandes gelten. Die vom Verstand zu unterscheidende Vernunft sei daher ihrem Inhalt nach als Inbegriff von Prinzipien unterbestimmt. Erst wenn beim Rückgang ins Prinzip auch zu einem Unbedingten übergegangen sei, gehöre das Prinzip dem Inhalt nach als solches der Vernunft an. Das mag sein. Aber dem Einwand wird im folgenden Rechnung getragen.
} 
Es liegt auf der Hand, daß damit die erwähnten Pendants zur Induktion und Abduktion noch kaum beachtet sind. ${ }^{3}$ Vor allem sind sie fürs Verfahren einer Bestimmung der Vernunft noch nicht ausgewertet. Nicht nur Kant, auch die meisten der nachkantischen Deutschen Idealisten geben uns davon keine rechte Idee. Die einzige Ausnahme ist Hegel. Sein Verfahren nämlich konkretisiert Kants Konzept einer zwischen Dogmatismus und Skeptizismus hindurchsteuernden, beide für sich arbeiten lassenden Untersuchung; zugleich aber berücksichtigt es die Forderungen, die sich aus den abstrakten Gegenstücken zur Induktion und Abduktion ergeben. Das ist nun kurz anzudeuten: In jedem Ganzen geregelter Schritte geht das Hegelische Verfahren aus von begrifflichen Elementen, an denen sich der dogmatische Verstand betätigt, $\{\mid$ S. 53\} während der Skeptiker an den Ergebnissen solcher Betätigung problematische, zu untersuchende Annahmen hat (oder sich deren bewußt wird). Diese Ausgangsaussagen stehen für den Dogmatiker in Verbindung mit der allgemeinen Voraussetzung fester Bestimmtheit der in ihnen enthaltenen Begriffe. Das macht das ,abstrakte oder verständige“ Moment jedes „Logisch-Reellen“ der Form nach aus (Enz., 79 f.). Der Skeptiker setzt dem — wie für das Gegenstück zur Induktion erforderlich — den Verdacht der Wahrheit einer prima vista besonders wenig plausiblen, allgemeinen Aussage entgegen: daß die feste Bestimmtheit, welche die Begriffe gegeneinander zu haben scheinen, keinen Bestand hat, sondern sich näher besehen von Fall zu Fall verlieren wird, — so daß die Frage entsteht, wie man begreifen kann, daß es sich so verhält. Dann werden — von den dogmatischen Aussagen ausgehend, wie es sich fürs Pendant zu einer Induktion gehört — beide einander entgegengesetzten, um Glaubwürdigkeit konkurrierenden Annahmen auf die Probe gestellt. Das ist die Phase des dialektischen oder negativ-vernünftigen Moments im Logisch-Reellen. In ihr stellt der Skeptiker bezüglich beider allgemeinen Annahmen - der skeptischen und der dogmatischen — seine Gedankenexperimente an, indem er die Annahmen in Beziehung auf ein jeweiliges ensemble von Begriffen erwägt. Er mobilisiert seinen ganzen Scharfsinn zu deduktiven Schlüssen aus dem vom Dogmatiker Angenommenen und trägt seine Äquipollenz-Attacken dagegen vor - wenn die kontra-intuitive Annahme des Skeptikers dabei bestärkt wird, mit dem Ergebnis, daß gegen die Aussagen des Dogmatikers (diejenige der allgemeinen Annahme fester Bestimmtheit der Begriffe eingeschlossen) mindestens ebenso starke Gründe sprechen wie für sie. Das Verfahren in dieser Phase erfüllt die umrissenen Forderungen eines Pendants zur Induktion: Es schafft optimale Bedingungen dafür, daß die Übergänge zu neuen Aussagen, wenn diese das jeweils erreichte Ergebnis berücksichtigen, auf das Wahre hin konvergieren. Für den Skeptiker aber endet das Verfahren jeweils in einem kontra-intuitiven , dem bleibt schrecklich viel schwer oder gar nicht Verträgliches ohne Wahrheitsanspruch nebeneinander stehen; aber für den dem Dogmatiker entgegengestellten Verdacht des Skeptikers ist Raum geschaffen.

Das ist der Ausgangspunkt für ein Pendant zur Abduktion, gegen das der Skeptiker keinen Einwand erheben kann: die Einführung eines neuen Begriffs, der spezifisch der Vernunft (und nicht nur dem Bedingtes denkenden Verstand) zuzuordnen ist — das spekulative oder positiv-vernünftige Moment jedes Logisch-Reellen. Der einzuführende Begriff muß so strukturiert sein, daß mit ihm das kontra-intuitive Phänomen der aufgedeckten Begriffsverhältnisse begreiflich wird, weil in ihm die Begriffe, die sich in diesen dubiosen Verhältnissen befinden, integriert sind und das Schwanken ihrer Bestimmtheit (wenn sie für sich genommen werden) plausibel

\footnotetext{
${ }^{3}$ Kant unterläßt es auch, diese Pendants zu beachten, wenn es um die Ableitung transzendentaler Ideen geht (KrV, A 334 ff.). Doch die Form deduktiver Vernunftschlüsse, auf deren Arten er in diesem Kontext verweist, taugt nicht alleine schon dazu, ,eine subjektive Anleitung“ für die Gewinnung solcher Ideen zu geben. Denn die Anleitung, die wir brauchen, darf nicht unterstellen, daß wir über die Prämissen einschlägiger Prosyllogismen und über die Begriffe für ihre „Exponenten“ (KrV, A 331) bereits verfügen. Sie muß uns vielmehr sagen, mit welcher Berechtigung wir zu ihnen gelangen können. Das ist nur möglich, wenn man den formellen Begriff der Vernunft in der skizzierten, von Peirce inspirierten Weise ergänzt.
} 
wird. Das Verfahren verbindet mit diesem neuen Begriff jedoch keine dogmatische Behauptung, sondern gibt ihm nur - der Forderung an die Abduktion entspre- $\{\mid$ S. 54 $\}$ chend — in der Liste möglicher Annahmen, die im weiteren zu prüfen sind, eine besonders hochrangige Stellung: der Begriff wird zur Grundlage für das, was es nun in einer neuen Einheit des ganzen Verfahrens zu prüfen und dann wieder aus der Prüfung an abduktiver Konsequenz zu ziehen gilt. Wie gefordert wird also eine gedankliche Kontinuität hergestellt, die das Suchen nach Wahrheit im jeweils erreichten Kontext vor chaotischer Instabilität bewahrt. In einer neuen Einheit des Verfahrens wird ja das ganze deduktive Potential der vorhergegangenen Verfahrensschritte einsetzbar und dem Skeptiker zur Disposition gestellt. Es wäre nicht schwer, auch zu zeigen, wie sich im Fortgang der Untersuchung ein System von Begriffen unter einem einzigen Begriff darstellt und daß dagegen der Skeptiker nicht mehr argumentieren kann, dieser Begriff sei entweder leer oder seine Erfülltheit zu behaupten sei dogmatisch. So ist also auch die Vernunft-Forderung erfüllbar, daß das Verfahren im fortgehenden Bestimmen (und nicht nur im Rückgang auf ein Allgemeinstes) bis zu einem Einzelnen komme und darin seinen Abschluß finde: dieses Einzelne ist die eine, nicht pluralisierbare Vernunft selbst. Hegels Ausdruck dafür ist „die Idee“ oder, genauer genommen, „die absolute Idee“. Diese Idee ist — in Kantisch-Fichtescher Perspektive gesprochen — die Vernunft selbst, sofern sie Subjekt-Objekt und als solches die Subjektivität des „Begriffs“ ist, der in der Objektivität zur Übereinstimmung mit sich gekommen ist, aber ebensosehr die Objektivität, die in sich (als innere Zweckmäßigkeit) zur Einheit des Begriffs mit sich zurückgekommen ist. Und sie ist zudem eine Idee,

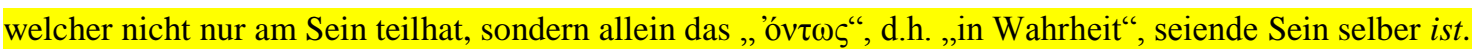

Was wäre von diesem Begriff der Vernunft aus zum Vernünftigen zu sagen, von dem meine Vorüberlegungen ausgegangen waren? Das auszumachen sollte die Aufgabe meines dritten Hauptkapitels (im ganzen also des fünften Kapitels) sein. Hier wenigstens noch ein paar Bemerkungen dazu.

\section{Zum Vernünftigen als solchen und in der Objektivität}

Gewiß: Nicht die Vernunft selbst ist vernünftig oder kann gar vernünftig sein, ohne es sein zu müssen. Nur etwas ist vernünftig, das sich in irgendeiner Hinsicht von der Vernunft selber auch unterscheidet, um in einer anderen Hinsicht an ihr partizipieren zu können, also „Anteil“ an ihr zu haben und darin wenigstens teilweise mit ihr übereinzustimmen. Aber dazu muß das Betreffende etwas anderes als Vernunft nicht so sehr sein, daß es vernünftig nur werden, vorübergehend sein und auch wieder zu sein aufhören könnte, wie $\mathrm{z}$. B. Zwecke und Mittel oder eine Form menschlichen Lebens. Im Gegenteil wird man wohl sagen müssen: Nur wenn zu demjenigen, was die Vernunft materialiter ausmacht, auch schon Vernünftiges gehört — sozusagen das Vernünftige als solches - hat man eine Chance, vom einen oder anderen dessen, was von der Vernunft, so genommen, unterschieden ist, also weiter von ihr entfernt, berechtigtermaßen zu sagen, es habe Vernunft oder auch: es sei $\{\mid$ S. 55\} (mehr oder weniger) vernünftig. Worin aber muß sich im Ganzen der Vernunft das gesuchte „Vernünftige als solches“ finden?

Ich glaube, man muß zur Beantwortung dieser Frage dem bereits Gesagten mindestens drei Ergänzungen hinzufügen (und hat in der dritten auch den Ansatzpunkt dafür, innerhalb der Auskunft über Vernunft von Objektivität und innerhalb der Auskunft über diese Objektivität von Zwecken und Mitteln sowie von ihrer Vernünftigkeit reden zu dürfen): 
1. Von der Idee als Subjekt-Objekt kann man sagen, sie sei schlechterdings das Vernünftige, solange sie nur als Idee überhaupt bestimmt ist, nicht aber als absolute Idee und schlechthinnige Singularität. Das Vernünftige, von dem hierbei die Rede ist, ist der Vernunft so nah, daß die Bedeutung des Adjektivs ,vernünftig“ in solcher Verwendung fast mit derjenigen des Substantivs ,Vernunft‘ zusammenfällt. ${ }^{4}$

2. Aufgrund der Rede von einem der Form nach negativ- und positiv-vernünftigen Moment eines jeden Logisch-Reellen läßt sich sagen: Was den begrifflichen Gehalt der Vernunft betrifft, gehört jeder dritte in der systematischen Aufeinanderfolge ihrer Begriffe zum in der Vernunft Vernünftigen seinem Inhalt nach. Jeder ist ein „Vernunftbegriff“ nicht nur in dem Sinn, daß er sich aus Vernunft rechtfertige, sondern auch im Sinne eines Begriffs vom in der Vernunft Vernünftigen. Allerdings kommt in jedem das Vernünftige der Vernunft nur partikularisiert zur Erkenntnis — nicht nur wegen der jeweils übrigen Vernunftbegriffe, sondern auch deshalb, weil in einem jeden die Bewegung, welche zur Vernunft als solcher gehört und ihre Form ausmacht, zur Ruhe gekommen ist. Eben darin hat dieses Vernünftige eine bloß teilweise Übereinstimmung mit der Vernunft.

3. Eine höhere Übereinstimmung und damit auch eine der Vernunft selbst nähere Weise des Vernünftigen als solchen wird somit wohl darin bestehen, daß in dem von einem solch dritten Begriff Begriffenen dessen Bewegung selbst schon enthalten ist: als diejenige wechselseitiger Vermittlung des Allgemeinen, Besonderen und Einzelnen in einem Schluß, welcher diese „Momente“ des Begriffs zu Einem zusammenschließt. In diesem anspruchsvollen Sinn der Rede vom an der Vernunft „teil“ habenden Vernünftigen ist nach Hegels Überzeugung der Schluß ,alles Vernünftige“ und alles Vernünftige in der Objektivität ein Schluß. Zum Begriff eines solchen Schlusses gehört, daß sich in seiner Vermittlung deren Bewegung am Ende aufhebt in ein ebenso Einzelnes wie Unmittelbares, aber durch besondere Allgemeinheit Bestimmtes. So kommt es mit dem Begriff eines Schlusses überhaupt erst zu jenem Begriff von Objektivität, den Kant mit Hilfe seines Konzepts von transzendentaler Apperzeption und von Urteilsform zu erreichen versucht, aber mit Hilfe dieser $\{\mid$ S. 56 $\}$ Konzepte allein (nebst Formen der Anschauung sowie Funktionen der Einbildungskraft) nur erschleichen kann. ${ }^{5}$ Hegel hingegen kann von hier aus die Objektivität nicht nur als Mechanismus, sondern auch als Teleologie, also als ein Verhältnis von Mitteln und Zwecken, denken - und kann die innere Teleologie als das in der gesamten Vernunft-Objektivität eigentlich Vernünftige begreifen. Wenn das Vernünftige diesseits von Zwecken und Mitteln so gedacht wird, wie Hegel es zu begreifen versuchte, läßt sich mithin nicht nur einsehen, wie Vernunft-Objektivität Teleologie einschließen kann, was immer unsere theoretische Erkenntnis vom Denken solcher Objektivität abhalten mag. Wir werden damit auch aus der unkomfortablen Alternative befreit, uns entweder (wie Spinoza) einem antiteleologischen Naturalismus verschreiben zu müssen, den unsere praktische Vernunft beständig dementiert, oder in einem philosophischen Dualismus beständig zu schwanken zwischen Denkforderungen der theoretischen und der praktischen Vernunft.

4 Vgl. Logik II, 173; Enz., § 214.

5 Denn Kants Deduktion reiner Verstandesbegriffe von einem Gegenstand möglicher Erkenntnis überhaupt bringt uns nicht zum Begriff von einem solchen Gegenstand als einem Einzelnen, obwohl es gerade dessen für die Anwendbarkeit von Kategorien bedürfte. Vgl. dazu Wildenauer, 2000, S. 28 ff. 


\section{Literaturverzeichnis}

Baumgarten, Alexander G. (1757): Metaphysica. Halle

Fulda, Hans F./Stolzenberg, Jürgen (Hrsg.) (2001): Architektonik und System in der Philosophie Kants. Hamburg.

Hegel, Georg W.F. (1981): Wissenschaft der Logik, Bd. 2. Die subjektive Logik (1816), in: Hegel, Georg W.F.: Gesammelte Werke, in Verbindung mit der Deutschen Forschungsgemeinschaft hrsg. v. d. RheinischWestfälischen Akademie der Wissenschaften, Bd. 12, hrsg. v. Hogemann, Friedrich, Hamburg. (Zitiert mit ,Logik II“ unter Angabe der Seitenzahl.)

Hegel, Georg W.F. (1992): Enzyklopädie der philosophischen Wissenschaften im Grundrisse (1830), in: Hegel, Georg W.F.: Gesammelte Werke, in Verbindung mit der Deutschen Forschungsgemeinschaft hrsg. v. d. Rheinisch-Westfälischen Akademie der Wissenschaften, Bd. 21, hrsg. v. Bonsiepen, Wolfgang, Hamburg. (Zitiert mit „Enz.“ unter Angabe des Paragraphen.)

Kant, Immanuel (1911): Kritik der reinen Vernunft, in: Kant's gesammelte Werke III/IV, hrsg. v. d. Königlich Preußischen Akademie der Wissenschaften, Berlin. (Zitiert mit „KrV“ unter Angabe der Seiten der ersten (A) und/oder zweiten (B) Originalausgabe.)

Wildenauer, Miriam (2000): Hegels logische Ideenlehre als Epistemologie eines freien Denkens, das praktisch werden kann. Freiheit als Schlußstein des Hegelschen Systems reiner, subjektiver und objektiver Vernunft, Heidelberg (Diss.). 